International Journal of Wireless \& Mobile Networks (IJWMN) Vol. 4, No. 4, August 2012

\title{
ANALYSIS OF A DETERMINISTIC JONG NANG GATE With TRANSMITTER COOPERATION
}

\author{
Moon Ho Lee ${ }^{1}$, Md. Hashem Ali Khan ${ }^{1}$ and Daechul Park ${ }^{2}$ \\ ${ }^{1}$ Division of Electronics \& Information Engineering, Chonbuk National University, \\ Jeonju 561-756, Korea \\ moonhodjbnu.ac.kr, hashem05aliejbnu.ac.kr \\ ${ }^{2}$ Dept. of Information \& Communication Engineering, Hannam University, Daedeok- \\ Gu, Daejeon 306-791, Korea \\ fia4joylyahoo.co.kr
}

\begin{abstract}
In this paper, we introduce practical the root of digital human binary coded Jong Nang communications as the wooden gate in Korea Jeju Island custom. We investigate Jong Nang gate models as an approximation of the AWGN model. The objective is to find a deterministic model, which is accessible to capacity analysis. Furthermore, this analysis should provide insights on the capacity of the AWGN model. Motivated by backhaul cooperation in cellular networks where cooperation is among base stations, we term the interference channel with conferencing transmitters. Jong Nang communications is normal 3 rafters placed on two vertical stones with three holes to convey the family's whereabouts that is deterministic signal, nowadays it is applied to backhaul in mobile base station.
\end{abstract}

\section{KEYWORDS}

Deterministic model, Jong Nang, Analysis of capacity \& Gaussian Interference channel

\section{INTRODUCTION}

Jong Nang, the wooden gate in Korea Jeju Island dialect, had three wooden rafters placed on Jong-Ju-Mok (two large vertical stones with three holes) to convey the family's whereabouts. A product of the wisdom of Jeju Island people in Korea, the Jong Nang was a unique custom of local culture. As there was no gate at the house in Jeju Island, timbers were used to prevent cattle or horses from entering and having the barley and millet that were spread out in the yard. Later, Jong Nang was developed into the means of informing visitors whether the residents were at home or not [1-6].

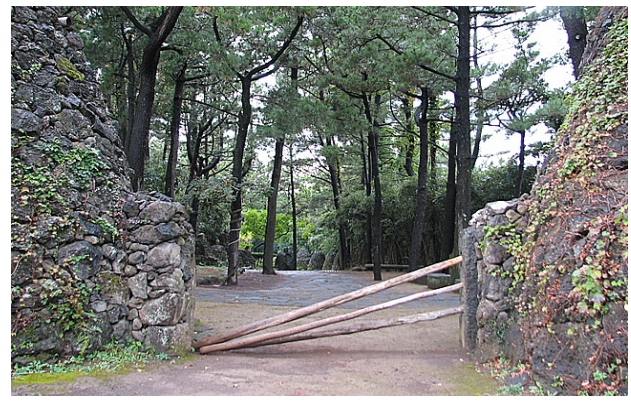

(a) 3 gate open $\left(\begin{array}{lll}0 & 0 & 0\end{array}\right)$

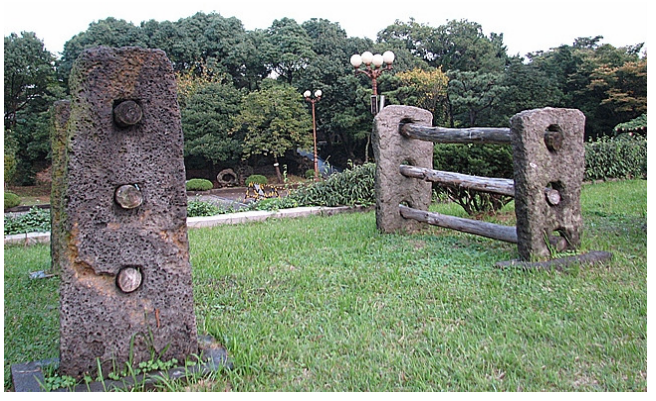

(b) 3 gate close ( $\left.\begin{array}{lll}1 & 1 & 1\end{array}\right)$

*This paper was partially presented at the WiMoN Conference, 13-15 July 2012, Chennai, India.

DOI : 10.5121/ijwmn.2012.4405 
The Jong Nang used the binary system similar to digital communications and computers today. Three timbers were exactly like three binary digits. The Jong Nang system could convey eight different messages. One of three Jong-Nang placed between the Jong-Ju-Mok, or "100" indicated there was no one at home, but the family would soon return form a neighboring area.

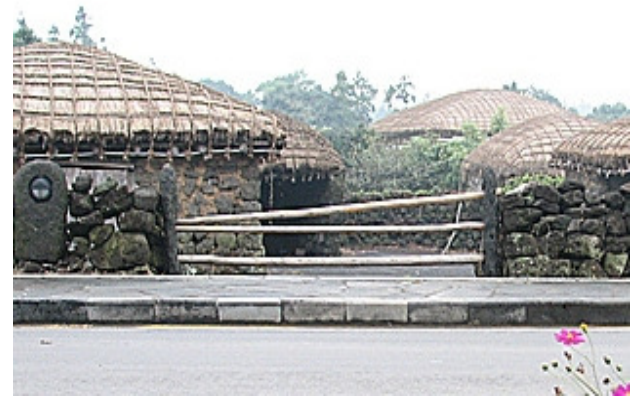

(c) 2 gate close: interference $\left(\begin{array}{lll}0 & 1 & 1\end{array}\right)$

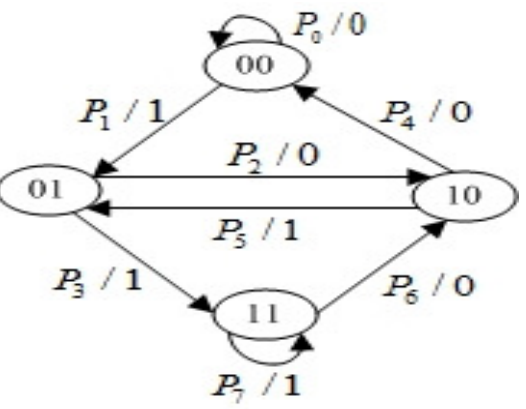

(d) Jong Nang Markov process

Figure 1. Korea Jeju Jong Nang

Two Jong Nangs or " 101 " meant the family was visiting a neighboring town and it would be a while before they returned. All these Jong Nangs or "111" announced the family was out of town for a long time, as shown in Fig 1. When none of the Jong Nang was placed, or "000", this meant the family was at home, as shown in Fig 1. This system derived from the life of the Jeju Island people. Table 1 shows the different Jong Nang messages. In paper [6] analysis of Jong Nang is one kind of practical deterministic scheme. Tse et al, Gamal \& other person have written many papers as deterministic model with Gaussian interference channel [9-14].

Table 1. The comparison of Decimal and Binary number in Jong Nang

\begin{tabular}{|c|c|c|c|}
\hline Decimal & Binary & Logic & Comparison \\
\hline 0 & 000 & $\vdots$ & Staying at home \\
\hline 1 & 001 & $\vdots$ & Visiting next door \\
\hline 2 & 010 & $\vdots$ & Visiting next door \\
\hline 3 & 011 & $\exists$ & Visiting neighbour \\
\hline 4 & 100 & $\vdots$ & Visiting next door \\
\hline 5 & 101 & $\exists$ & Visiting neighbour \\
\hline 6 & 110 & $\exists$ & Visiting neighbour \\
\hline 7 & 111 & $\exists$ & Long time out of home \\
\hline
\end{tabular}

From Table 1, binary MSB (Most significant bit) are 00,00,01,01, 10,10,11,11 and LSB (Least significant bit) are $0,1,0,1,0,1,0,1$. The probability $\left(p_{0} \cdots p_{7}\right)$ of Table 2 is similar as Fig $1(\mathrm{~d})$. This process is Jong Nang Markov chains. Table 2 shows Markov state of the Jong Nang 3NOR gate based on Table 1 . The daily life of householder is staying or out of house based on Table 1.

Table 2. Markov state of Jong Nang 3-input NOR gate

\begin{tabular}{|l|l|l|l|l|l|}
\hline Decimal & Binary & Probability & Decimal & Binary & Probability \\
\hline 0 & 000 & $\mathrm{P}_{0}$ & 4 & 100 & $\mathrm{P}_{4}$ \\
1 & 001 & $\mathrm{P}_{1}$ & 5 & 101 & $\mathrm{P}_{5}$ \\
\hline
\end{tabular}


International Journal of Wireless \& Mobile Networks (IJWMN) Vol. 4, No. 4, August 2012

\begin{tabular}{|l|l|l|l|l|l|}
\hline 2 & 010 & $\mathrm{P}_{2}$ & 6 & 110 & $\mathrm{P}_{6}$ \\
3 & 011 & $\mathrm{P}_{3}$ & 7 & 111 & $\mathrm{P}_{7}$ \\
\hline
\end{tabular}

The main motivation of this paper based on $[6,9,10]$ AWGN capacity by Jong Nang gate modelling in Figure 2. The Jong Nang gate (JNG) is practical system and comparison Gaussian interference channel (IC).The JNG network is exact analysed and we get approximate analysis results. The deterministic model is defined in a way that allows for exact analysis of its capacity as Han-Kobayashi.

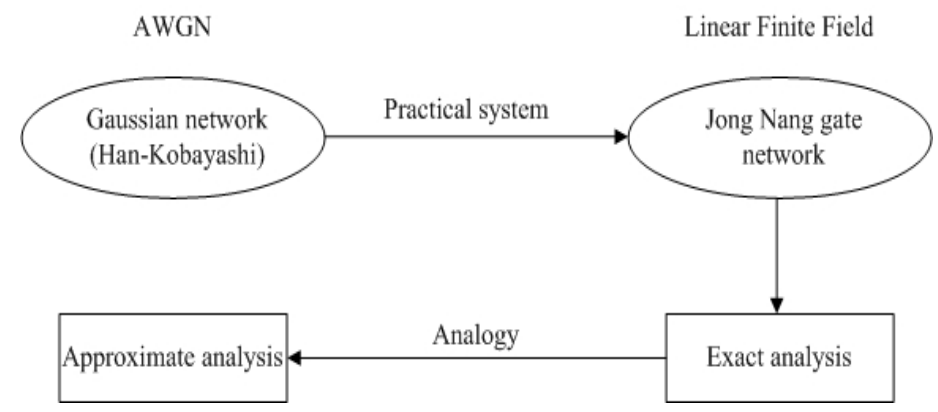

Figure 2. AWGN capacity by the Jong Nang gate modelling

The approximation of the Gaussian Interference channel (IC) by the q-ary expansion deterministic channel (QED) was first proposed by Avestimehr, Diggavi, and Tse. Bresler, Parekh, and Tse applied this approach to approximate the many-to-one Gaussian IC. The symmetric capacity achieving linear coding scheme for q-ary expansion deterministic IC is due to Jafar and Vishwanath. Bandermer showed that the entire capacity region can be achieved by this linear coding scheme. In this paper, we also introduce Jong Nang deterministic channel approach to transmitter's cooperation.

This paper is organized as follows. Section 2 describes practical system of Jong Nang gate model. Section 3 presents capacity of the Jong Nang channel as 3-input NOR channel. Section 4 presents the Jong Nang binary erasure multiple access channel. Section 5 JNG of Gaussian IC. Jong Nang gate interference channel with transmitter's cooperation and conclusions are delivered in Section 6 \& 7 .

\section{Practical System of Jong Nang Gate Model}

In this section, we introduce the practical system of JNG model in Figure 2. The idea deterministic modelling was may be most clearly explained by Tse [10]. The idea is to define a deterministic model in such a way that each AWGN network has a corresponding representation in the JNG model based on Table 1. The deterministic model is defined in a way that allows for exact analysis of its capacity. In the last step we hope to derive approximate results for the AWGN network by using the exact analysis from the deterministic network [9].

We might define any deterministic model and find the corresponding representation of our AWGN network. The two challenges are depicted in the lower half of the figure. Firstly, we have to analyse the capacity in the JNG model. Secondly, we have to show that the deterministic capacity is an approximation to the AWGN capacity in some sense. 
International Journal of Wireless \& Mobile Networks (IJWMN) Vol. 4, No. 4, August 2012

Table 3. Backhaul

\begin{tabular}{|c|c|c|c|c|}
\hline Technology & Max. Bandwidth & Min. node latency & Link latency & Max. length \\
\hline Ethernet & $100 \mathrm{Gbit} / \mathrm{s}$ & $\mathrm{A} \mathrm{few}^{3} \mu \mathrm{s}$ & $5 \mu \mathrm{s} / \mathrm{km}$ (fiber) & $40 \mathrm{~km}$ \\
AGPONI & $10 \mathrm{Gbit} / \mathrm{s}$ shared & $\sqcup 100 \mu \mathrm{s} \mathrm{(upstr.)}$ & $5 \mu \mathrm{s} / \mathrm{km}$ (fiber) & $20 \mathrm{~km}$ \\
DVSL2 & Mult $^{\mathrm{b}} .100 \mathrm{Mbit} / \mathrm{s}$ & $1.25 \ldots 2 \mathrm{~ms}^{\mathrm{c}}$ & $<$ node latency & $\sqcup 0.4 \mathrm{~km}^{\mathrm{d}}$ \\
Microware & $1 \mathrm{Gbit} / \mathrm{s}$ & $\sqcup 100 \mu \mathrm{s}$ & $3 \mu \mathrm{s} / \mathrm{km}($ air) & $\mathrm{A} \mathrm{few}^{\mathrm{e}} \mathrm{km}$ \\
\hline
\end{tabular}

A very much simplified model will offer simple capacity analysis, but the approximation result might be difficult to find or might not hold, because the two models are rather unlike. On the other hand, a JNG model that is similar to the AWGN model might offer a shortcut to the approximation result, but finding its capacity might not be much simpler than the original problem. Now a day CoMP Backhaul is important for communications. We investigate limited transmitter or receiver cooperation helps mitigate interference. The behaviour of the benefit brought by transmitter cooperation is the same as by receiver cooperation. Backhauling for a CoMP enabled radio access network (RAN) therefore requires new backhaul technologies as shown in Table 3.

\section{Capacity of The Jong Nang Channel as 3-InPUt NOR Channel}

When the Jong Nang channel is viewed as a coordinated 3-input noiseless multiple access NOR channel with the following transition probabilities: $p(y=1 \mid x=100)=p(y=0 \mid x=100)=$ $p(y=0 \mid x=101)=p(y=0 \mid x=111)=1$, the capacity of the channel can be calculated $[7,8]$

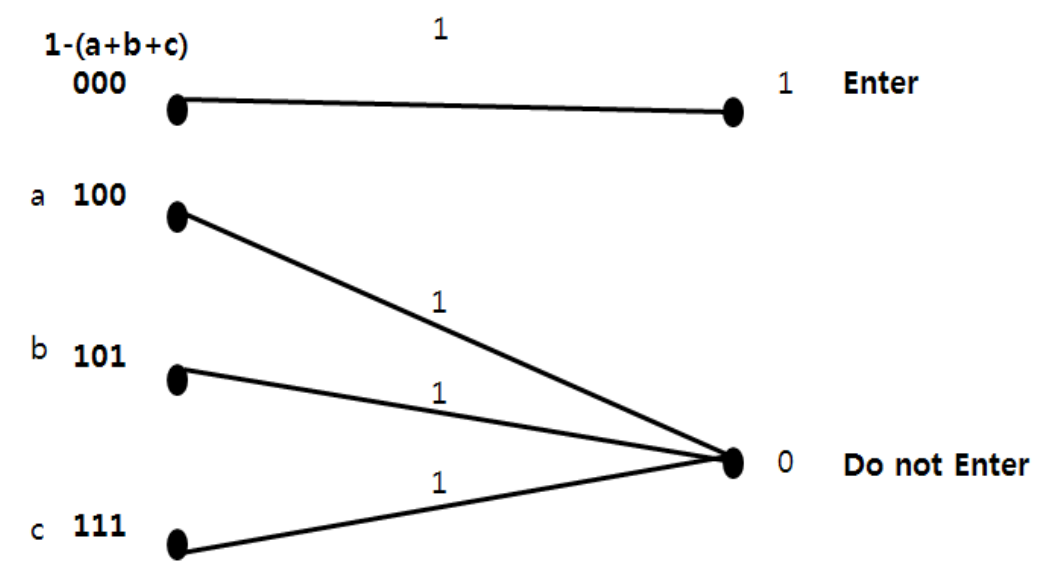

Figure 3. The Jong Nang channel

Theorem: The capacity of the Jong Nang Channel modelled as 3-input noiseless NOR channel is one.

Proof: Let $p(x=100)=a, p(x=101)=b, p(x=111)=c$, and $p(x=000)=1-(a+b+c)$. The output of the channel then has the following distribution:

$$
p(y=0)=a+b+c, \quad p(y=1)=1-(a+b+c)
$$

Furthermore, we can calculate the joint distributions:

$$
p(y=1, x=000)=p(y=1 \mid x=000) p(x=000)=1-(a+b+c)
$$


International Journal of Wireless \& Mobile Networks (IJWMN) Vol. 4, No. 4, August 2012

$$
\begin{aligned}
& p(y=0, x=100)=p(y=0 \mid x=100) p(x=100)=a \\
& p(y=0, x=101)=p(y=0 \mid x=101) p(x=101)=b \\
& p(y=0, x=111)=p(y=0 \mid x=111) p(x=111)=c
\end{aligned}
$$

Now, to compute the mutual information between the input and the output of the channel $I(X ; Y)=H(Y)-H(Y \mid X)$, we evaluate $H(Y)$ and $H(Y \mid X)$. Let $q=a+b+c$, we have

$$
\begin{gathered}
H(Y)=-(1-q) \log (1-q)-q \log (q)=h(q) \\
H(Y \mid X)=-(1-q) \log (1)-a \log (1)-b \log (1)-c \log (1)=0
\end{gathered}
$$

Hence,

$$
I(X ; Y)=H(Y)-H(Y \mid X)=h(q)
$$

Maximizing the mutual information with respect to $q$,

$$
\ln (2) d I(X ; Y) / d q=1+\ln (1-q)-1-\ln (q)=\ln ((1-q) / q)=0
$$

which implies that $q=1 / 2$.

Therefore, the capacity of the channel is $h(1 / 2)=1$. QED.

That the capacity of the channel is 1 and can be achieved when $q=1 / 2$ implies that the family can communicate at most one bit of information per three timbers using this channel, with the requirement that the family is home half of the time.

\section{The Jong Nang Binary Erasure Multiple Access Channel}

We can see that the Jong Nang code does not have any error. In the case that the channel has noise, which may result from a timber falling down from its place by natural or manmade sort of event, an error detecting code maybe desired. As shown Figure 3 4:

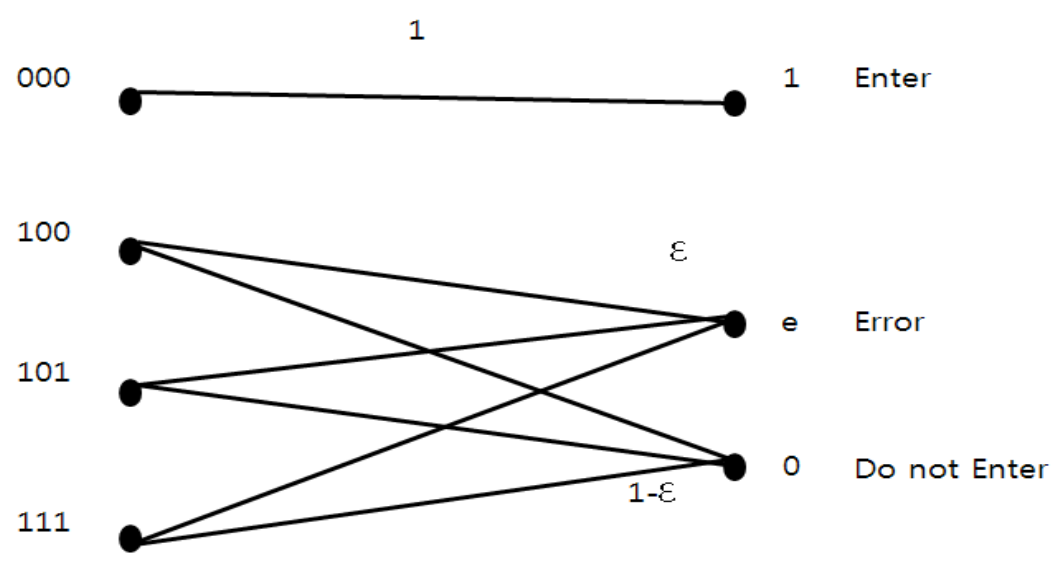

Figure 4. The Jong Nang binary erasure channel 
International Journal of Wireless \& Mobile Networks (IJWMN) Vol. 4, No. 4, August 2012

As we mentioned, the channel does not take care the error number. It only takes whether the error is occurred or not. So all errors can be detected by using the codes, but the number of errors cannot be detected. In the following analysis, we assume that errors can occur when timbers fall down from its original place.

As shown Fig 5, the occured error includes all case of the errors; first, we transmit '111' which has the message 'out of town' and noise also occured, and we receive '101' which has the message 'visiting neighboring Village', this channel is transmitting the wrong message by ocuuring the error. Second, we transmit '111' which has the defined message and noise also occured. We receive ' 110 ' which has the undefined message.

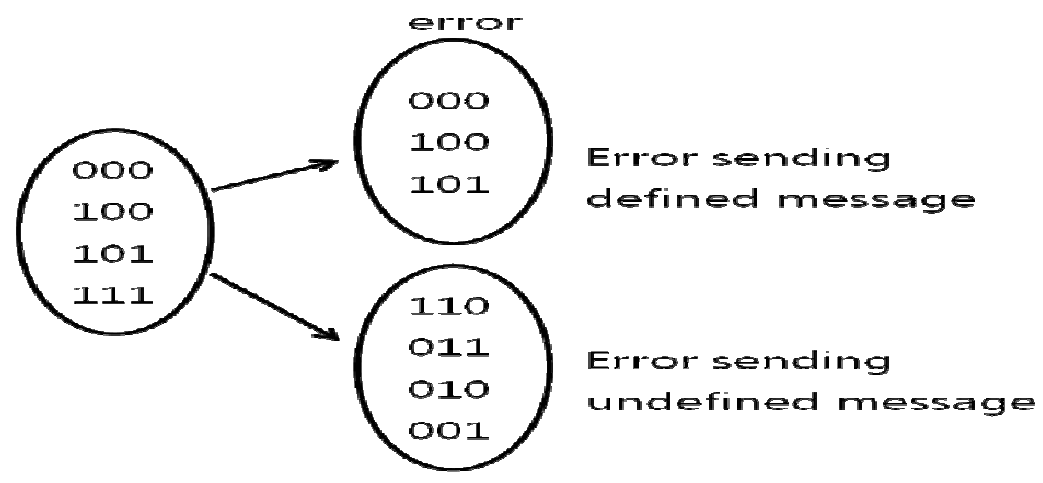

Figure 5. The event diagram of the occured error

Consequently, the only allowed words are 000, 011, 101, 110, 001, 010, 100. Furthermore, to reflect the situation in which the error is detect, the "erased" channel output is created, and let probabilities that an erasure occurs given channel inputs 000, 100, 101 and 111 are the same and equal to $\varepsilon$. That is when a visitor sees $110,001,010$ or 001 , channel output is set to "erased." Under these conditions, the channel transition probabilities are given,

$$
\begin{gathered}
p(y=1 \mid x=000)=1 \\
p(y=0 \mid x=011)=p(y=0 \mid x=101)=p(y=0 \mid x=110)=1-\varepsilon \\
p(y=e \mid x=011)=p(y=e \mid x=101)=p(y=e \mid x=110)=\varepsilon
\end{gathered}
$$

Theorem: The capacity of the Noisy Jong Nang Channel as described above is 1 .

Proof: Let $p(x=000)=a, p(x=100)=b, p(x=101)=c, p(x=111)=1-(a+b+c)$, and $q=a+b+c$. The output of the channel then has the following distribution:

$$
\begin{gathered}
p(y=1)=1-(a+b+c)=1-q, p(y=e)=\varepsilon(a+b+c)=\varepsilon q \\
p(y=0)=(1-\varepsilon)(a+b+c)=(1-\varepsilon) q
\end{gathered}
$$

Furthermore, we can calculate the joint distributions:

$$
\begin{aligned}
& p(y=1, x=000)=p(y=1 \mid x=000) p(x=000)=1-(a+b+c) \\
& p(y=0, x=100)=p(y=0 \mid x=100) p(x=100)=a(1-\varepsilon) \\
& p(y=0, x=101)=p(y=0 \mid x=101) p(x=101)=b(1-\varepsilon)
\end{aligned}
$$


International Journal of Wireless \& Mobile Networks (IJWMN) Vol. 4, No. 4, August 2012

$$
\begin{gathered}
p(y=0, x=111)=p(y=0 \mid x=111) p(x=111)=c(1-\varepsilon) \\
p(y=e, x=100)=p(y=e \mid x=100) p(x=100)=a \varepsilon \\
p(y=e, x=101)=p(y=e \mid x=101) p(x=101)=b \varepsilon \\
p(y=e, x=111)=p(y=e \mid x=111) p(x=111)=c \varepsilon
\end{gathered}
$$

Now, to compute the mutual information between the input and the output of the channel $I(X ; Y)=H(Y)-H(Y \mid X)$, we evaluate $H(Y)$ and $H(Y \mid X)$.

The capacity of the channel $(C)$ is maximum of the mutual information; that is, when $H(Y \mid X)=0$, we find the capacity of the channel.

Let $q=a+b+c$, we have

$$
\begin{aligned}
& H(Y)=-(1-q) \log (1-q)-\varepsilon q \log \varepsilon q-(1-\varepsilon) q \log (1-\varepsilon) q \\
& H(Y \mid X)=-(1-q) \log (1)-\varepsilon q \log \varepsilon-(1-\varepsilon) q \log (1-\varepsilon)
\end{aligned}
$$

Hence,

$$
\begin{array}{r}
I(X ; Y)=H(Y)-H(Y \mid X)=-(1-q) \log (1-q)-\varepsilon q \log \varepsilon q-(1-\varepsilon) q \log (1-\varepsilon) q \\
+(1-q) \log (1)+\varepsilon q \log \varepsilon+(1-\varepsilon) q \log (1-\varepsilon)
\end{array}
$$

Maximizing the mutual information with respect to $q, \ln (2) d I(X ; Y) / d q$

$$
\begin{aligned}
& =1+\ln (1-q)-\varepsilon-\varepsilon \ln \varepsilon q-(1-\varepsilon)-(1-\varepsilon) \ln (1-\varepsilon) q+\varepsilon+\ln \varepsilon+(1-\varepsilon) \ln (1-\varepsilon) \\
& =\ln (1-q)-\varepsilon \ln q-(1-\varepsilon) \ln q=\mathrm{n}(1-q)-\ln q=\ln [(1-q) / q]=0
\end{aligned}
$$

which implies that $q=1 / 2$.

Therefore, the capacity of the channel is

$$
\begin{aligned}
C & =-0.5 \log (0.5)-0.5 \varepsilon \log 0.5 \varepsilon-0.5(1-\varepsilon) \log 0.5(1-\varepsilon)+0.5 \varepsilon \log \varepsilon+0.5(1-\varepsilon) \\
\log (1-\varepsilon) & =-0.5 \log 0.5+0.5 \varepsilon \log 2+0.5(1-\varepsilon) \log 2=0.5+0.5=1 . \text { QED. }
\end{aligned}
$$

We can analysis capacity of the Jong Nang Binary Erasure multiple access channel (MAC), in which four senders send the information to 3 receivers. This channel has binary inputs, $X_{1}, X_{2} \in\{000,100,101,111\}$ and a ternary output $Y_{e}, Y_{1}, Y_{0}$.

Proof: Note that $p(x=000)=p(x=100)=p(x=101)=p(x=111)=1 / 4$. We can represent $000,101,110$ and 111 by $00,10,01,11$ respectively. For the give information $X_{1}, X_{2} \in\{000,100,101,111\}$ and ternary outputs $Y \in\{1, e, 0\}$. We can get the channel capacity

$$
C_{1}<I\left(X_{1} ; Y \mid X_{2}\right), \quad C_{2}<I\left(X_{2} ; Y \mid X_{1}\right)
$$

We also get

$$
C_{1}=C_{2}=\operatorname{Max}\left\{I\left(X_{1} ; Y \mid X_{2}\right)\right\}
$$


International Journal of Wireless \& Mobile Networks (IJWMN) Vol. 4, No. 4, August 2012

$$
=\sum_{X_{1}} \sum_{X_{2}} \sum_{Y} p\left(X_{1}\right) p\left(X_{2}\right) p\left(Y \mid X_{1}, X_{2}\right) \log \frac{p\left(Y \mid X_{1}, X_{2}\right)}{\sum_{X_{1}} p\left(X_{1}\right) p\left(Y \mid X_{1}, X_{2}\right)}
$$

$$
=1 \text {, as shown in Figure 6(a). }
$$

Then the channel capacity of the combined channel is

$$
\begin{aligned}
C_{12} & =\operatorname{Max} I\left(X_{1}, X_{2} ; Y\right) \\
& =\sum_{X_{1}} \sum_{X_{2}} \sum_{Y} p\left(X_{1}\right) p\left(X_{2}\right) p\left(Y \mid X_{1}, X_{2}\right) \log \frac{p\left(Y \mid X_{1}, X_{2}\right)}{\sum_{X_{1}} \sum_{X_{2}} p\left(X_{1}\right) p\left(X_{2}\right) p\left(Y \mid X_{1}, X_{2}\right)}
\end{aligned}
$$

It is easy to see that

$$
\begin{gathered}
P(Y=1)=P(X=000)=K, P(Y=\varepsilon)=P(X=100) \varepsilon+P(X=101) \varepsilon+P(X=111) \varepsilon \\
=[P(X=000)+P(X=101)+P(X=111)] \varepsilon=3 K \varepsilon, \text { and } \\
P(Y=0)=[P(X=000)+P(X=101)+P(X=111)](1-\varepsilon)=3 K(1-\varepsilon)
\end{gathered}
$$

Consequently,

$$
H(Y)=-[K \log K+3 K \varepsilon \log 3 K \varepsilon+3 K(1-\varepsilon) \log K(1-\varepsilon)]
$$

Since $K=1 / 4, \varepsilon=1 / 2$ and $H\left(Y \mid X_{1}, X_{2}\right)=0$, we have

$$
H(Y)=-\left[\frac{1}{4} \log \frac{1}{4}+3 \times \frac{1}{4} \times \frac{1}{2} \log \frac{3}{8}+3 \times \frac{1}{4} \times \frac{1}{2} \log \frac{3}{8}\right]=1.56
$$

and $\operatorname{Max}\left(C_{12}\right) \approx 1.56$, which is shown in Figure 6(b).

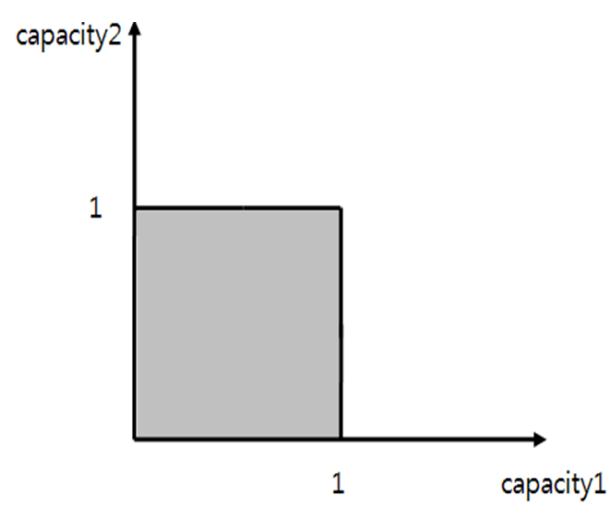

(a) Independent

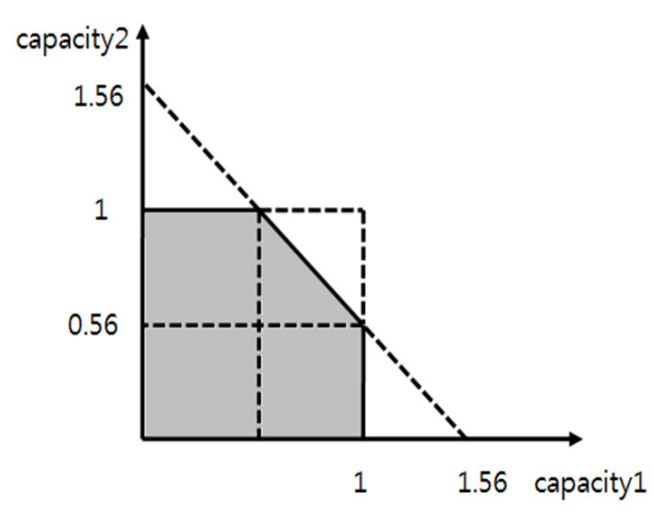

(b) Dependent

Figure 6. Capacity region for the Jong Nang channels

In case Figure 6(a), the inputs of Jong Nang Erasure multiple access channel are independent, there is no interference between the senders. In case Figure 6(b), the inputs of Jong Nang channel are dependent, there is interference between the senders. 


\section{Jong Nang Gate of Gaussian IC}

We introduce the q-ary expansion JNG interference channel and it closely the Gaussian IC in the limit of high SNR. Consider the symmetric case where the interference is specified by the parameter $\alpha \in[0,2]$ and Table 1 . Figure 7 shows JNG \& Gaussian interference channel. We express $X_{1}$ as $\left[x_{1, L-1}, x_{1, L-2,} x_{1, L-3}, \cdots x_{1,0}\right]$ and similarly for $X_{2}$.

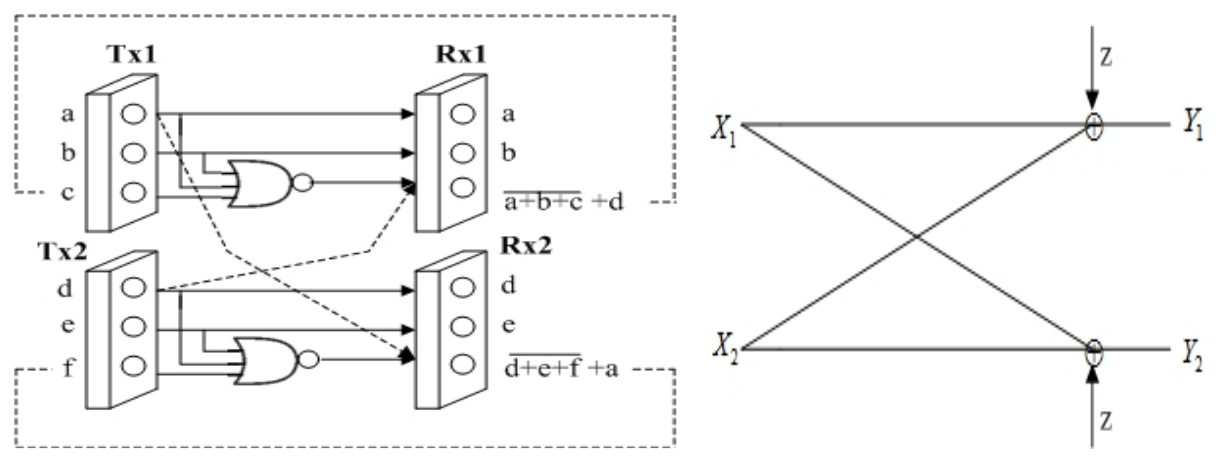

Figure 7. Block diagram of (a) JNG \& (b) Gaussian IC

The JNG rafter up and down the output of the channel depends on whether the shift is positive or negative. The shift parameter is defined by

$$
s=(\alpha-1) L,
$$

where $L$ is $q$ ary digit number. Now we get shift parameter from Eq. (1),

$$
s=(\alpha-1) L=(1 / 3-1) 6=-4 .
$$

where $\alpha=1 / 3, L=6$ from Fig 7(a).

\subsection{Downshift}

Here $0 \leq \alpha \leq 1, Y_{1}$ is a q-ary

$$
Y_{1}=\left\{\begin{array}{l}
a \\
a+b+c \\
\end{array} d(\bmod q)\right.
$$

The outputs of the channel is represented as

$$
Y_{1}=X_{1}+G_{s} X_{2}
$$

where $G_{s}$ is a shift matrix.

\subsection{Upshift}

Here $1 \leq \alpha \leq 2$,

$$
Y_{2}=\left\{\begin{array}{l}
\frac{d}{d+e+f}+a(\bmod q) \\
c
\end{array}\right.
$$


The outputs of the channel is represented as

$$
Y_{2}=G_{s} X_{1}+X_{2},
$$

The capacity region of the symmetric QED-IC can be obtained by a straightforward of the capacity of the injective deterministic IC. The normalized capacity region is the set of rate pairs $\left(R_{1}, R_{2}\right)$ such that

$$
\begin{aligned}
& R_{1} \leq 1, R_{2} \leq 1, \\
& R_{1}+R_{2} \leq \max \{2 \alpha, 2-2 \alpha\} \\
& R_{1}+R_{2} \leq \max \{\alpha, 2-\alpha\}
\end{aligned}
$$

for $\alpha \in[1 / 2,1]$, and

$$
\begin{aligned}
& R_{1} \leq 1, R_{2} \leq 1 \\
& R_{1}+R_{2} \leq \max \{2 \alpha, 2-2 \alpha\} \\
& R_{1}+R_{2} \leq \max \{\alpha, 2-\alpha\}
\end{aligned}
$$

for $\alpha \in[0,1 / 2]$.

The capacity region of the symmetric QED-IC can be achieved error free using a single linear coding scheme. For encoding, we use the matrix $A$. We get $[A]_{6}$ from Figure 7 (a). Consider a binary expansion deterministic IC with $q=2, L=6$, and $\alpha=1 / 3$.

$$
[A]_{6}=\left[\begin{array}{cccccc}
1 & 0 & 0 & 0 & 0 & 1 \\
0 & 1 & 0 & 0 & 0 & 0 \\
0 & 0 & 1 & 0 & 0 & 0 \\
0 & 0 & 1 & 1 & 0 & 0 \\
0 & 0 & 0 & 0 & 1 & 0 \\
0 & 0 & 0 & 0 & 0 & 1
\end{array}\right] \bmod 2
$$

This decoding procedure corresponds to multiplying the output by the matrix $B$.

$$
[B]_{6}=\left[\begin{array}{cccccc}
1 & 0 & 0 & 0 & 0 & -1 \\
0 & 1 & 0 & 0 & 0 & 0 \\
0 & 0 & 1 & 0 & 0 & 0 \\
0 & 0 & -1 & 1 & 0 & 0 \\
0 & 0 & 0 & 0 & 1 & 0 \\
0 & 0 & 0 & 0 & 0 & 1
\end{array}\right] .
$$

Note that $[B]_{6}[A]_{6}=[I]_{6}$ and $B G_{s} A=0$, hence interference is cancelled while the intended signal is recovered perfectly. The transmits signal, $X_{j}=A U_{j}$, where $A$ is q-ary matrix and $U_{j}$ is uniformly distributed set of binary vectors. Decoder $j$ multiplies its received symbol $Y_{j}$ by a corresponding matrix $B$ to recover $U_{j}$ perfectly. For decoding case, $Y_{j}=B U_{j}$.

For encoding case,

$$
X_{1}=A U_{1}, X_{2}=A U_{2}
$$

and decoding case,

$$
Y_{1}=B U_{1}, Y_{2}=B U_{2}
$$


International Journal of Wireless \& Mobile Networks (IJWMN) Vol. 4, No. 4, August 2012

The transmitted symbol $X_{j}$ and the received vector $Y_{j}$ are shown in Figure 8. Decoding for $U_{1}$ can be performed as follows:

$$
\begin{gathered}
U_{1,0}=Y_{1,0}, U_{1,1}=Y_{1,1}, U_{1,4}=Y_{1,4}, U_{1,5}=Y_{1,5} \\
U_{1,3}=Y_{1,3} \oplus Y_{1,4}=Y_{1,3} \oplus U_{2,5} \text { and } U_{1,6}=Y_{1,6} \oplus Y_{1,1}=Y_{1,6} \oplus U_{2,2}
\end{gathered}
$$

The symmetric capacity is expressed as

$$
C_{s y m}=H\left(U_{j}\right)=I\left(U_{j} ; Y_{j}\right)=I\left(X_{j} ; Y_{j}\right)
$$

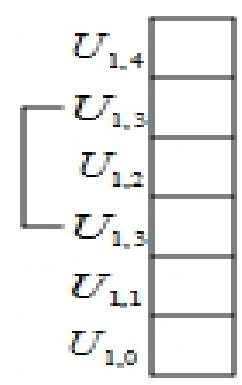

$X_{2}$

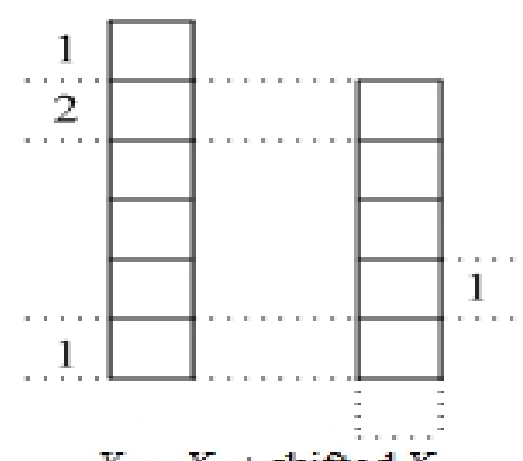

$Y_{1}=X_{1}+$ shifted $X_{2}$

Figure 8. Transmitted symbol $X_{j}$ and the received vector $Y_{j}$

\section{Jong NANG Gate IC COOPERATION TRANSMitTERS}

In this section, we discuss Jong Nang gate interference channel with cooperation transmitters, to overcome the complications both in achievability and inner bounds. The corresponding linear deterministic channel is parameterized by nonnegative integers $n_{11}, n_{21}, n_{22}, n_{12}, k_{12}$ and $k_{21}$, where $n_{i j}=\left(\left\lfloor\log \left|h_{i j}\right|^{2}\right\rfloor\right)^{+}, i, j=\{1,2\}$.

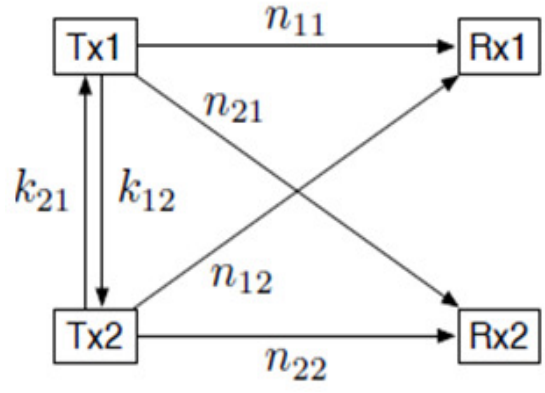

(a) Channel model

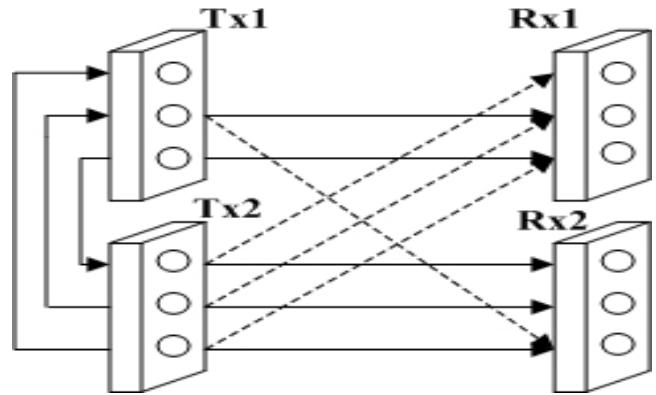

(b) Cooperation transmitters Jong Nang gate

Figure 9. Jong Nang gate IC with cooperation transmitters

Figure 9 shows Jong Nang gate with cooperation transmitters. A natural cooperative strategy between transmitters is that, prior to each block of transmission, two transmitters hold a 
International Journal of Wireless \& Mobile Networks (IJWMN) Vol. 4, No. 4, August 2012

conference to tell each other part of their messages [12]. Hence the messages are classified into two kinds: (1) cooperative messages, which are those known to both transmitters due to the conference, and (2) noncooperative ones, which are those unknown to the other transmitter since the cooperative link capacities are finite.

The generalization comes from making the mappings from $X_{1}$ to $T_{1}$ and from $X_{2}$ to $T_{2}$ in Figure 10.

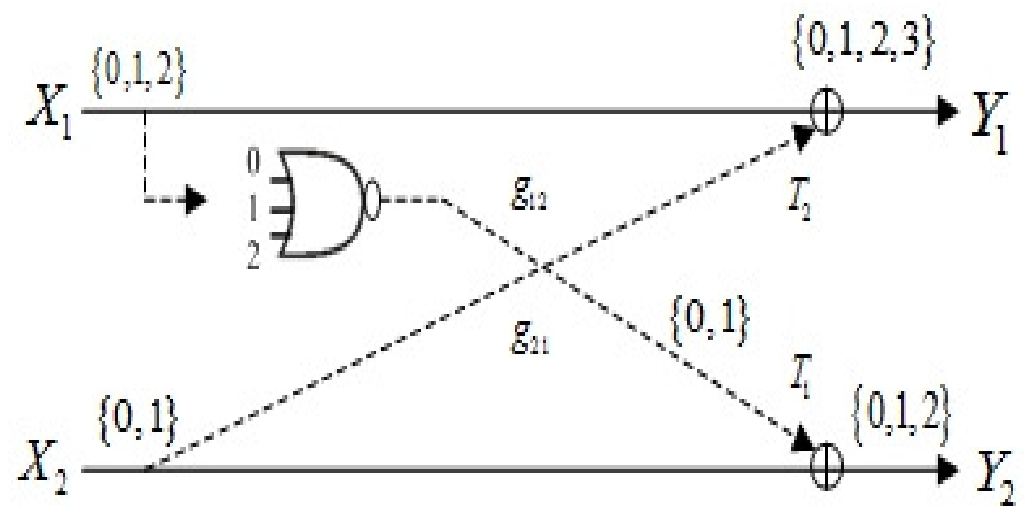

Figure 10. Block diagram of interference channel

The channel outputs are given by

$$
Y_{1}=y_{1}\left(X_{1}, T_{2}\right), Y_{2}=y_{2}\left(X_{2}, T_{1}\right)
$$

where $T_{1}=t_{1}\left(X_{1}\right), T_{2}=t_{2}\left(X_{2}\right), X_{1}=\left\{x_{10}, x_{11}, x_{12}\right\}$, and $X_{2}=\left\{x_{20}, x_{21}, x_{22}\right\}$. We assume the functions $y_{1}$ and $y_{2}$ are injective in $t_{1}$ and $t_{2}$ respectively. We imply that $H\left(Y_{1} \mid X_{1}\right)=H\left(T_{2}\right)$ and $H\left(Y_{2} \mid X_{2}\right)=H\left(T_{1}\right)$. The probability of two transmitters are $P\left(T_{1} \mid x_{1,0}, x_{1,1}, x_{1,2}\right)$ and $P\left(T_{2} \mid x_{2,0}, x_{2,1}, x_{2,2}\right)$.

The Han-Kobayashi inner bound with the restriction that $p\left(u_{1}, u_{2} \mid q, x_{1}, x_{2}\right)=p_{T_{1} \mid x_{1}}\left(u_{1} \mid x_{1}\right)$ $p_{T_{2} \mid x_{2}}\left(u_{2} \mid x_{2}\right)$ reduces to the following [13].

Proposition: Any rate pair $\left(R_{1}, R_{2}\right)$ for the Jong Nang deterministic IC must satisfy the inequalities

$$
\begin{gathered}
R_{1} \leq H\left(Y_{1} \mid U_{2}, Q\right)-H\left(T_{2} \mid U_{2}, Q\right), \\
R_{2} \leq H\left(Y_{2} \mid U_{1}, Q\right)-H\left(T_{1} \mid U_{1}, Q\right),
\end{gathered}
$$

We specialize the inner bounds to Gaussian as follows:

\subsection{Time division}

We obtain the time division inner bound on the capacity region of Gaussian IC such that

$$
R_{1}<\alpha C\left(S N R_{1} / \alpha\right), R_{2}<\bar{\alpha} C\left(S N R_{2} / \bar{\alpha}\right) \text { for } \alpha \in[0,1]
$$




\subsection{Treating interference as noise}

For treating interference as noise case, the capacity of region

$$
R_{1}<C\left(\frac{S N R_{1}}{1+I N R_{1}}\right), \quad R_{2}<C\left(\frac{S N R_{2}}{1+I N R_{2}}\right)
$$

\subsection{Interference decoding}

The capacity region of the Gaussian IC with strong interference is the set of rate pairs such that

$$
\begin{aligned}
& R_{1} \leq C\left(S N R_{1}\right), R_{2} \leq C\left(S N R_{2}\right) \\
& R_{1}+R_{2} \leq \min \left\{C\left(S N R_{1}+I N R_{1}\right), C\left(S N R_{2}+I N R_{2}\right)\right\}
\end{aligned}
$$

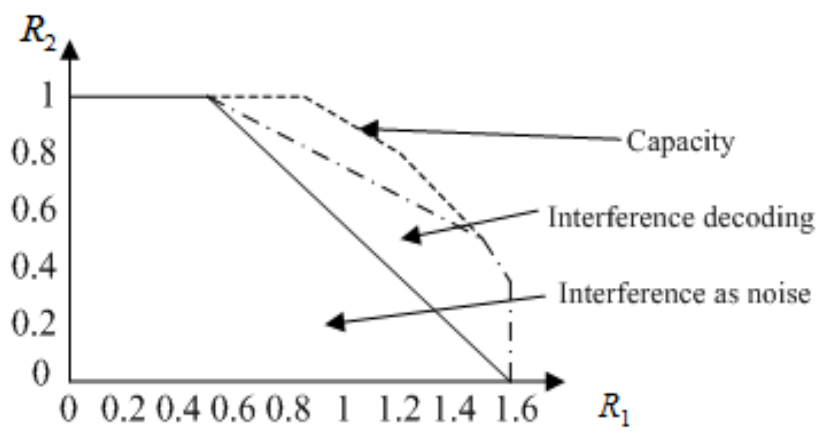

Figure 11. Capacity region and inner bounds

The above inner bounds are compared in Figure 11. It compares this inner bound to the capacity region given in [14] and to the region achievable by treating interference as noise. Interference decoding does not achieve the full capacity. Han-Kobayashi rate splitting and superposition coding are needed for full capacity. Finally, the combining functions and receiver functions are taken to be addition.

\subsection{Feedback case}

The scheme uses two stages. In the first stage, transmitter 1 and $2 \operatorname{send}(a, b, c)$ and $(d, e, f)$ respectively. Each receiver defers decoding to the second stage. In the second stage, using feedback, each transmitter decodes information of the other user: transmitter 1 and 2 decode $(d, e, f)$ and $(a, b, c)$, respectively. Each transmitter then sends information of the other user. Now each receiver can decode its own data from two received signals. Notice that the second stage was used for refining all bits sent previously, without sending additional information. In this case, we consider $c$ and $f$. When we get output is one then feedback possible. Our logic is calculated in Table 4. 
Table 4. Feedback case

\begin{tabular}{|c|c|c|c|c|c|c|c|c|c|c|c|}
\hline \multicolumn{3}{|c|}{ Txl Input } & \multicolumn{3}{c|}{ Tx2 Input } & \multicolumn{3}{c|}{ Rxl Output } & \multicolumn{3}{c|}{ Rx2 Output } \\
\hline a & b & c & d & e & f & a & b & s & d & e & w \\
\hline 0 & 0 & 0 & 1 & 1 & 1 & 0 & 0 & 0 & 1 & 1 & 0 \\
\hline 0 & 0 & 1 & 1 & 1 & 0 & 0 & 0 & 1 & 1 & 1 & 0 \\
\hline 0 & 1 & 0 & 1 & 0 & 1 & 0 & 1 & 1 & 1 & 0 & 0 \\
\hline 0 & 1 & 1 & 1 & 0 & 0 & 0 & 1 & 1 & 1 & 0 & 0 \\
\hline 1 & 1 & 1 & 0 & 0 & 0 & 1 & 1 & 0 & 0 & 0 & 0 \\
\hline
\end{tabular}

\section{Conclusions}

In this paper, we investigate the achievable symmetric rate for the Jong Nang gate interference channel. The deterministic models can be utilized to approximate the capacity of wireless communication networks and to design transmission strategies. The capacity of the JNG which means the wooden gate in Korea Jeju island dialect, had three wooden rafters placed on JongJu-Mok (two large vertical stones with three holes) to convey the family's whereabouts was calculated. It is shown that this interference-decoding inner bound is tight under certain strong interference conditions. The inner bound is also shown to strictly contain the inner bound obtained by treating interference as noise, which includes interference alignment for deterministic channels. Practical JNG will be reasonable well matched to Tse [10] deterministic model of Gaussian IC.

\section{ACKNOWLEDGEMENT}

This work was supported by the World Class University (WCU) R32-2012-000-20014-0, BSRP 2010-0020942, MEST 2012-002521, NRF, Korea, the 2011 Korea-China International Cooperative Research Project (Grant No D00066, I00026) and ${ }^{2}$ Hannam University while taking a sabbatical leave in SRM University, India.

\section{REFERENCES}

[1] M. H. Lee, “Jong Nang (正木 )”, EXPO '93 Information \& Telecom. Pavilion poster, 1993.

[2] M. H. Lee, The History of Information and Communication, Kimyeong-Sa, Seoul, 1994.

[3] M. H. Lee, "Jong Nang: The symbol of digital communication and Ying and Yang" Telecom, vol. 9 , no. $1,1993$.

[4] M. H. Lee, “Jong Nang System”, Patent, no. 133285, Korea, 1998.

[5] M. H. Lee, "The History of Jeju Jong Nang Binary Code”, IEEE VTS News, vol. 50, no. 1, 2003.

[6] M. H. Lee, X. Jiang, C. H. Choe, S. H. Kim, "Analysis of Jong Nang Multiple Access Channel', ISITA 2006, Seoul, Korea, 2006.

[7] T. M. Cover, J. A. Thomas, Elements of Information Theory, John Wiley \& Sons, New York, 1991.

[8] C. E. Shannon, "A Mathematical Theory of Communication”, Bell System Technical Journal, vol. 27, pp. 379-423 and 623-656, 1948.

[9] N. Schrammar, “On Deterministic Models for Wireless Networks", Licentiate Thesis in Telecommunications Stockholm, Sweden 2011.

[10] A. Salman, D. Suhas, D. Tse, “A Deterministic approaches to wireless relay networks", ISIT, 2007. 
International Journal of Wireless \& Mobile Networks (IJWMN) Vol. 4, No. 4, August 2012

[11] C. Suh, D. Tse, "Symmetric feedback capacity of the Gaussian interference channel to within one bit”, ISIT 2009, Seoul, Korea, 2009.

[12] I. H. Wang, D. Tse, "Interference mitigation through limited transmitter cooperation", IEEE Trans. on Information Theory, vol. 57, no. 5, May 2011.

[13] A. El Gamal, Y. H. Kim, Network Information Theory, Cambridge University Press, 2011.

[14] B. Bandemer, A. El Gamal, "Interference decoding for deterministic channels", IEEE Trans. Information Theory, vol. 57, no. 5, May 2011.

Moon Ho Lee is a professor and former chair of the Department of Electronics Engineering in Chonbuk National University, Korea. He received the Ph.D. degree from Chonnam National University, Korea in 1984, and from the University of Tokyo, Japan in 1990, both Electrical Engineering, He was in University of Minnesota, U.S.A, from 1985 to 1986 as a post-doctor. He has been working in Namyang MBC broadcasting with chief engineer from 1970 to 1980, after then he joined to Chonbuk National University as a Professor. Dr. Lee has made significant original contributions in the areas of mobile communication

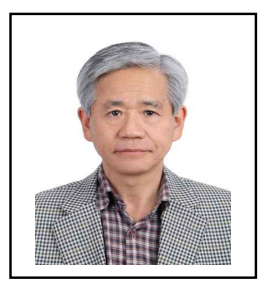
code design, channel coding, and multi-dimensional source and channel coding. He has authored 34 books, 155 SCI papers in international journals, and 240 papers in domestic journals, and delivered 350 papers at international conferences. Dr. Lee is a member of the National Academy of Engineering in Korea and a Foreign Fellow of the Bulgaria Academy of Sciences. He is the inventor of Jacket Matrix and it in Wikipedia was cited over 65,363 times, July 20, 2012.

Md. Hashem Ali Khan received his B.S. (Hons) and M.S. degree in Applied Physics, Electronics, and Communication Engineering from Islamic University in Bangladesh in 2003 and 2004, respectively. He is currently doing research towards Ph.D. degree of Electronic Engineering as a researcher at the Institute of Information and Communication in Chonbuk National University, Republic of Korea, since 2009. His current research areas are MIMO, OFDM, and Jacket Matrices.

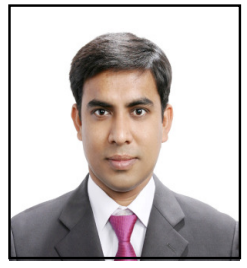

Daechul Park is a professor and former chair of the Department of Electronics Engineering and former director of Engineering Accreditation Innovation Center, in Hannam University, Korea. He graduated Sogang University, Seoul, Korea in 1977. He received the MS and Ph.D. degree from the University of New Mexico, Albuquerque, NM, USA in 1985 and 1989, respectively. During 1977-1982, he worked in ADD (Agency for Defense Development) as a research engineer. While studying in USA, he worked as a research assistant in computer vision lab

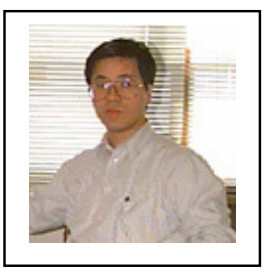
in UNM. Since he finished his doctoral degree in 1989, he joined ETRI (Electronics and Telecommunication Research Institute as a senior technical staff and conducted several research projects until 1993. During 1991-1992, he carried out joint international project with Prof. Martin Vetterli, Center for Telecommunication, Columbia University, NY, USA. Dr. Park moved his position to Hannam University in 1993 and conducted several national projects as a leading researcher. He spent a sabbatical leave in the University of Texas, Arlington, TX, U.S.A, from 2000 to 2001 as a visiting professor. Currently, Dr. Park is in Dept. of ECE, SRM University, Kattankulathur, Tamilnadu, India. Dr. Park has made original contributions in the areas of digital image processing and coding, signal processing algorithm development, digital television standard, and communication code design. He has authored 7 books, many papers in international journals, and more than 30 papers in domestic journals Dr. Park is a member of the professional Engineering Societies in Korea and serves as a board member of The Korea Institute of Signal Processing and Systems. 